\title{
RANDOM SAMPLING OF TRIVIALS WORDS IN FINITELY PRESENTED GROUPS
}

\author{
M. ELDER, A. RECHNITZER, AND E. J. JANSE VAN RENSBURG
}

\begin{abstract}
We describe a novel algorithm for random sampling of freely reduced words equal to the identity in a finitely presented group. The algorithm is based on Metropolis Monte Carlo sampling. The algorithm samples from a stretched Boltzmann distribution

$$
\pi(w)=(|w|+1)^{\alpha} \beta^{|w|} \cdot Z^{-1}
$$

where $|w|$ is the length of a word $w, \alpha$ and $\beta$ are parameters of the algorithm, and $Z$ is a normalising constant. It follows that words of the same length are sampled with the same probability. The distribution can be expressed in terms of the cogrowth series of the group, which then allows us to relate statistical properties of words sampled by the algorithm to the cogrowth of the group, and hence its amenability.

We have implemented the algorithm and applied it to several group presentations including the Baumslag-Solitar groups, some free products studied by Kouksov, a finitely presented amenable group that is not subexponentially amenable (based on the basilica group), and Richard Thompson's group F.
\end{abstract}

\section{INTRODUCTION}

In this article we propose a new random sampling algorithm for finitely presented groups. The algorithm samples freely reduced words in the generators that are equal to the identity of the group. This algorithm is based on ideas from statistical mechanics and Markov chain theory. In particular, the algorithm is inspired by the BFACF algorithm for sampling self-avoiding polygons (we refer the reader to [20, 26] for a description of BFACF and self-avoiding polygons). The algorithm differs from previous work on random walks in groups in that it only samples trivial words. Indeed, it can be seen as executing a random walk on the space of trivial words, rather than a random walk on the Cayley graph of the group.

We prove that the algorithm coverges to a specified distribution, and relate this distribution to the cogrowth series of the group. By varying a parameter, we can detect numerically the precise position of the radius of converge of the cogrowth series, and hence numerically predict the amenability or non-amenability of the group.

We have implemented the algorithm and have applied it to a selection of finitely presentated groups. These include several Baumslag-Solitar groups, some free products whose cogrowth series were studied by Kouksov [23, a finitely presented relative of the basilica group, and R. Thompson's group $F$.

Date: November 14, 2018.

2010 Mathematics Subject Classification. 20F69, 20F65, 05A15, 60J20.

Key words and phrases. Cogrowth; amenable group; Metropolis algorithm; Baumslag-Solitar group; R. Thompson's group $F$. 
The present article continues previous work by the authors [15, 16, where various techniques, also based in statistical mechanics and enumerative combinatorics, were applied to the problem of estimating and computing the cogrowth of groups. This in turn built on previous work of Burillo, Cleary and Wiest [6], and Arzhantseva, Guba, Lustig, and Préaux [1, who applied experimental techniques to the problem of deciding the amenability of Thompson's group $F$. In other work, Belk and Brown 5 proved the currently best known upper bound for the isoperimetric constant for $F$, and Moore 30 gives lower bounds on the growth rate of Følner function for $F$.

More generally a (by no means exhaustive) list of others working in the area of random walks on groups is Bartholdi [2, 3, 4, Diaconis and Saloff-Coste [10, 11, 12, 8, 9], Dykema [13, 14, Lalley [24, Smirnova-Nagnibeda [31, 32] and Woess [33, 37].

For the benefit of readers outside of group theory, and to establish notation, we start with a precise definition of group presentations and cogrowth.

Definition 1.1 (Presentations and trivial words). A presentation

$$
\left\langle a_{1}, \ldots, a_{k} \mid R_{1}, \ldots, R_{\ell}, \ldots\right\rangle
$$

encodes a (finitely generated) group as follows.

- Let $\mathcal{S}=\left\{a_{1}, a_{1}^{-1}, \ldots, a_{k}, a_{k}^{-1}\right\}$ be a set of $2 k$ distinct letters, and $\mathcal{S}^{*}$ the set of all finite strings or words over the letters in $\mathcal{S}$.

- A word in $\mathcal{S}^{*}$ is called freely reduced if it contains no subword of the form $a_{i} a_{i}^{-1}$ or $a_{i}^{-1} a_{i}$ for any $a_{i} \in \mathcal{S}$.

- The set of all freely reduced words, together with the operation of concatenation followed by free reduction (deleting $a_{i} a_{i}^{-1}$ and $a_{i}^{-1} a_{i}$ pairs) forms a group, called the free group on the letters $\left\{a_{1}, \ldots, a_{k}\right\}$, which we denote by $F\left(a_{1}, \ldots, a_{k}\right)$.

- Let $R_{1}, \ldots, R_{\ell}, \ldots$ be a finite or infinite list of distinct words in $F\left(a_{1}, \ldots, a_{k}\right)$.

- Let $N\left(R_{1}, \ldots, R_{\ell}, \ldots\right)$ be the normal subgroup of the free group consisting of all words of the form $\prod_{j=1}^{m} \rho_{j} R_{j} \rho_{j}^{-1}$ after free reduction, where $\rho_{i}$ is any element in the free group, and $R_{j}$ is one of the relators or their inverses. This subgroup is called the normal closure of the set of relators.

- The group encoded by the presentation $\left\langle a_{1}, \ldots, a_{k} \mid R_{1}, \ldots, R_{\ell}, \ldots\right\rangle$ is defined to be the quotient group $F\left(a_{1}, \ldots, a_{k}\right) / N\left(R_{1}, \ldots, R_{\ell}, \ldots\right)$.

- The letters $a_{i}$ are called generators, and the words $R_{i}$ are called relations or relators.

- A group $G$ is called finitely generated if it can be encoded by a presentation with the list $a_{1}, \ldots, a_{k}$ finite, and finitely presented if it can be encoded by a presentation with both lists $a_{1}, \ldots, a_{k}$ and $R_{1}, \ldots, R_{\ell}$ finite. In this article the list $a_{1}, \ldots, a_{k}$ will always be finite.

- It follows that a word in $F\left(a_{1}, \ldots, a_{k}\right)$ equals the identity element in $G$ if and only if it lies in the normal subgroup $N\left(R_{1}, \ldots, R_{\ell}, \ldots\right)$, and so is equal to a product of conjugates of relators and their inverses.

We will make extensive use of this last point in the work below. We call a word in $F\left(a_{1}, \ldots, a_{k}\right)$ that equals the identity element in $G$ a trivial word.

Let $c(n)$ be the number of freely reduced words, $w \in \mathcal{S}^{*}$, of length $n$ that represent the identity of a finitely generated group. This function is called the cogrowth function and the corresponding generating function is called the cogrowth 
series. The rate of exponential growth of the cogrowth function is the cogrowth of the group (with respect to a chosen finite generating set). Equivalently the cogrowth is the reciprocal of the radius of convergence of the cogrowth series. Grigorchuk and independently Cohen [7, 18, proved that a finitely generated group is amenable if and only if its cogrowth is $|\mathcal{S}|-1$.

For more background on amenability and cogrowth see [27, 36. The free group on two (or more) letters, as defined above, is known to be non-amenable. Also, subgroups of amenable groups are also amenable. It follows that if a group contains a subgroup isomorphic to the free group on 2 generators $\left(F\left(a_{1}, a_{2}\right)\right.$ above), then it cannot be amenable.

It is important to note that in some cases the letters in $\mathcal{S}$ may represent the same group element, for example, consider the presentation $\left\langle a \mid a^{2}\right\rangle$, where the relation $a^{2}$ implies that $a=a^{-1}$. In this example $|\mathcal{S}|=2$ (the letters $a, a^{-1}$ are considered distinct formal symbols), and the cogrowth function is $c(0)=1, c(2 n)=$ $2, c(2 n+1)=0$. The cogrowth series is then

$$
\sum c(n) z^{n}=1+2 z^{2}+2 z^{4}+\cdots=\frac{1+z^{2}}{1-z^{2}},
$$

and one can see directly that the radius of convergence is $1=|\mathcal{S}|-1$. Note that Kouksov 22] showed that a group has rational cogrowth series if and only if it is finite.

The article is organised as follows. In Section 2 we describe the algorithm for sampling trivial words from a given finite presentation. We then analyse the algorithm and show that it samples from a stretched Boltzmann distribution (Corollary 2.13). In Section 3 we apply the algorithm to several finite presentations. In cases where the cogrowth series is known, we see excellent agreement between the exact results and numerical data generated by our algorithm (for both amenable and non-amenable groups). We also apply the algorithm to sample words from groups for which the cogrowth series is not known, including Thompson's group $F$. We summmarise our results in Section 4 .

\section{Metropolis Sampling of Freely Reduced Trivial Words in Groups}

Let $G=\left\langle a_{1}, \ldots a_{k} \mid R_{1}, \ldots, R_{\ell}\right\rangle$ be a finitely presented group, and let $\mathcal{X}$ be the set of all freely reduced trivial words in $G$. We assume that the words $R_{i}$ are freely reduced and non-empty. Define a set $\mathcal{R}$ as follows. Take all the relators $R_{i}$, their inverses $R_{i}^{-1}$, and all cyclic permutations of these. The set $\mathcal{R}$ consists of all of these words after free reduction. For example, in the case of $\operatorname{BS}(2,3)=\left\langle a, b \mid a^{2} b a^{-3} b^{-1}\right\rangle$ the single relator yeilds $2 \times 7=14$ elements in $\mathcal{R}$.

We will describe an algorithm which samples a sequence of freely reduced trivial words

$$
\left(w_{0}, w_{1}, w_{2}, \ldots, w_{n}, \ldots\right) ; \quad w_{i} \in \mathcal{X} .
$$

We refer to the words $w_{i}$ as states. The algorithm constructs a new state $w_{n+1}$ from the current state $w_{n}$ by applying one of two elementary moves with specified probabilities that depend only on $w_{n}$. Such a procedure is known as a Markov chain.

There are two parts to the selection rule in the Markov chain - the elementary moves which transform $w_{n}$ to $w_{n+1}$ and the probabilities with which they are implemented. The implementation we use is known as Metropolis sampling [28]. In 
this way our algorithm is a Metropolis algorithm sampling along a Markov chain in $\mathcal{X}$.

2.1. Elementary moves. In this subsection we describe several elementary moves that we will perform on words in $\mathcal{X}$ to obtain other words in $\mathcal{X}$. Our goal is to define a set of moves that have a well defined reverse move, and such that any two words in $\mathcal{X}$ are connected by a finite sequence of moves.

The moves we describe are all based on the following two operations: conjugation by $x \in \mathcal{S}$; and insertion of $R \in \mathcal{R}$. For technical reasons which will describe below, we consider only what we call left-insertions rather than arbitrary insertions of relators. The elementary moves are as follows.

On input $w \in \mathcal{X}$ :

- (Conjugation by $x$ ) Let $x \in \mathcal{S}$. Write $w^{\prime}=x w x^{-1}$ and perform free reductions on $w^{\prime}$ to produce $w^{\prime \prime}$. Return $w^{\prime \prime}$.

- (Left-insertion of $R$ at position $m$ ) Let $R \in \mathcal{R}$ and $m \in\{0,1, \ldots,|w|\}$. Partition $w$ into two subwords $u$ and $v$, with $|v|=m$. Form $w^{\prime}=u R v$, and freely reduce this word by first freely reducing $u R$, obtaining $u^{\prime} v$, and then freely reducing to obtain $w^{\prime \prime}$. If $m=0$, then $R$ is appended to $w$, and if $m=|w|$, then $R$ is prepended to $w$.

Return $w^{\prime \prime}$ unless a symbol of $v$ is cancelled during the free-reduction step (i.e. a cancellation occurs to the right of $R$ ). If this occurs then we set $w^{\prime \prime}=w$ and return $w^{\prime \prime}$ (and so return a copy of the original word $w$ ).

Note that conjugations change word length by at most 2, and left-insertions by at most $|R|$.

Since $|\mathcal{S}|,|\mathcal{R}|$ and words $w \in \mathcal{X}$ are all finite, there are finitely many possible elementary moves from a state $w$ to a state $u$. The next two lemmas show that elementary moves are "uniquely reversible" in the sense that if there are $p$ conjugations and $q$ left-insertions from a state $w$ to a state $z$, then the same number of each type send $z$ to $w$.

For example, if $R=a b c \in \mathcal{R}, w=a b c a b c a b c$ and $z=a b c a b c a b c a b c$, there are exactly 4 left-insertions of $R$ possible in $w$ to obtain $z$, and exactly 4 left-insertions in $z$ to get $w$.

Lemma 2.1. Let $w, z \in \mathcal{X}$ with $w \neq z$. If $z$ is obtained from $w$ by a conjugation move, then either:

- there is exactly one conjugation move from $w$ to $z$, and exactly one conjugation move from $z$ to $w$; or

- there are exactly two conjugation moves from $w$ to $z$, and exactly two conjugation moves from $z$ to $w$. In this case $w=(x y)^{n}$ and $z=(y x)^{n}$ for some $x, y \in \mathcal{S}$.

Proof. Suppose $x, y$ are distinct symbols in $\mathcal{S}$, and $z$ is obtained from $w$ by conjugation by either $x$ or $y$.

- If $x w x^{-1}$ is freely reduced, then $y w y^{-1}$ must freely reduce to $z=x w x^{-1}$, and since both words have the same length, they must be identical and $x, y$ are the same symbol.

- If $w=x^{-1} w_{1}$ and $w_{1} x^{-1}$ is freely reduced, then $y x^{-1} w_{1} y$ must freely reduce to $z=w_{1} x^{-1}$, so must contain a cancellation. If $y x^{-1}$ is a free reduction then $x$ and $y$ are the same symbol, so the cancellation must be in $w_{1} y$, 
so $w_{1}=w_{2} y^{-1}$. So $z=w_{2} y x^{-1}=y x^{-1} w_{2}$ and the two expressions are identical strings, so $w_{2}$ must be a product of $\left(y x^{-1}\right)$ pairs, so $w=\left(y x^{-1}\right)^{n}$. In this case we have exactly two conjugations from $\left(y x^{-1}\right)^{n}$ to $\left(x^{-1} y\right)^{n}$, and exactly two back the other way (namely conjugation by $x^{-1}$ or $y^{-1}$ ).

- If $w=w_{1} x$ and $x w_{1}$ is freely reduced, then $y w_{1} x y^{-1}$ must freely reduce to $z=x w_{1}$ so contains a cancellation. Since $x, y$ are assumed distinct the cancellation must be in $y w_{1}$, so $w_{1}=y^{-1} w_{2}$ and $w_{2} x y^{-1}=x y^{-1} w_{2}$ are identical strings, so $w=\left(x y^{-1}\right)^{n}$ and we have exactly two conjugations from $\left(x y^{-1}\right)^{n}$ to $\left(y^{-1} x\right)^{n}$ and back.

- If $w=x^{-1} w_{1} x$ then $y x^{-1} w_{1} x y^{-1}$ must freely reduce to $z=w_{1}$, so since $x^{-1} w_{1} x$ is freely reduced (it is in $\mathcal{X}$ ) we must have $x, y$ are the same symbol.

Lemma 2.2. Let $w, z \in \mathcal{X}$ with $w \neq z$. If $w \rightarrow z$ by insertion of $R \in \mathcal{R}$ at position $m$, then $z \rightarrow w$ by insertion of $R^{-1} \in \mathcal{R}$ at position $m$.

Proof. Let $w=u v$ with $|v|=m$. If $u R v$ is not freely reduced then we have $u=u_{1} u_{2}, R=u_{2}^{-1} r$, and $u_{1} r$ is freely reduced. Then $w=u_{1} u_{2} v, z=u_{1} r v$. Note that by definition there is no cancellation of the suffix $v$.

Then left-inserting $R^{-1}$ at position $m$ in $z$ gives $u_{1} R^{-1} v=u_{1} r r^{-1} u_{2} v=u_{1} u_{2} v=$ $w$.

Note that for arbitrary insertions of relators, the previous lemma does not hold. For example consider the group $\mathbb{Z}^{2}=\left\langle a, b \mid b a b^{-1} a^{-1}\right\rangle$ and let $w=a^{3} b^{4} a^{-1} b^{-1} a b a^{-4} b^{-4}$ and $z=a^{4} b^{4} a^{-3} b^{-4}$. Inserting the relator $R=b a b^{-1} a^{-1}$ into $w$ at $m=9$ gives

$$
\begin{aligned}
a^{3} b^{4} a^{-1} b^{-1} \cdot R \cdot a b a^{-3} b^{-4} & \longrightarrow a^{3} b^{4} a^{-1} b^{-1} \cdot b a b^{-1} a^{-1} \cdot a b a^{-3} b^{-4} \\
& \longrightarrow a^{3} b^{3} a^{-1} \cdot a b a^{-3} b^{-4} \\
& \longrightarrow a^{3} b^{3} b a^{-3} b^{-4}
\end{aligned}
$$

This move is not a left-insertion since there is cancellation to the right of the inserted relator. Suppose it were allowed. Then there is no way to obtain $w$ via insertion of $R^{-1}=a b a^{-1} b^{-1}$ at any position in $z$, as one can easily verify by trying each position. By restricting to only left-insertions we avoid such problems, and guarantee that elementary moves have well defined reverse moves.

Lemma 2.3. Let $G, \mathcal{S}, \mathcal{R}, \mathcal{X}$ be as above. Let $w \in \mathcal{X}$ then there exists a finite sequence of conjugations and left-insertions that transform the empty word to $w$.

Proof. A word $u \in\left\{a_{1}^{ \pm 1}, \ldots, a_{k}^{ \pm 1}\right\}^{*}$ represents the identity element in $G$ if and only if it is the product of conjugates of the relators $R_{i}^{ \pm 1}$. So since $w \in \mathcal{X}$, it can be written as the product

$$
\prod_{j=1}^{n} \rho_{j} r_{j} \rho_{j}^{-1}
$$

after free reduction, where $\rho_{j} \in \mathcal{S}^{*}$ and $r_{j}=R_{i_{j}}^{ \pm 1}$.

We can obtain $w$ using conjugation and left-insertion as follows:

- set $u$ to be the empty word;

- left-insert $r_{1}$ after which $u=r_{1}$;

- conjugate by $\rho_{2}^{-1} \rho_{1}$ one letter at a time to obtain $u=\rho_{2}^{-1} \rho_{1} r_{1} \rho_{1}^{-1} \rho_{2}$ after free reduction; 
- left-insert $r_{2}$ at the extreme right $(m=0)$;

- repeat the previous two steps (conjugating by $\rho_{j+1}^{-1} \rho_{j}$ then left-inserting $r_{j}$ at the extreme right) until $r_{n}$ is left-inserted at the extreme right;

- conjugate by $\rho_{n}$.

Since we only ever append $r_{j}$ to the extreme right of the word, there are no right cancellations.

Note that since conjugations and left-insertions are reversible it follows that given any two words in $\mathcal{X}$ there is some finite sequence of elementary moves that transforms one to the other.

The reader may find it useful to consider the set $\mathcal{X}$ of states as the vertices of a graph, with states connected by directed edges if there is an elementary move from one to another, labeled by $(\operatorname{conj}, x)$ if it is conjugation by $x \in \mathcal{S}$, and (insert, $R, m$ ) if it is a left-insertion of $R \in \mathcal{R}$ at position $m \in \mathbb{N}$. The above lemmas prove that each edge between distinct states has a unique corresponding reverse edge with appropriate label, and that the graph is connected.

2.2. Transition probabilities. In this subsection we define probabilities with which elementary moves are selected or rejected.

Let $p_{c} \in(0,1), \alpha \in \mathbb{R}$ and $\beta \in(0,1)$ be parameters of the algorithm. Fix a probability distribution, $P$, over $\mathcal{R}$, so that $P(R)$ is the probability of choosing $R \in \mathcal{R}$. Further, assume that $P(R)>0$ for all $R \in \mathcal{R}$ and also that $P(R)=P\left(R^{-1}\right)$. Since $\mathcal{R}$ is finite, the obvious choice of $P$ is the uniform distribution - indeed this is what we used in our implementation. The algorithm we describe can easily be modified for presentations with infinitely many relators by choosing an appropriate distribution on $\mathcal{R}$ in this case - see subsection 2.7 below.

Let $w_{n}$ be the current word. We construct the next word, $w_{n+1}$ as follows:

- With probability $p_{c}$ choose to perform a conjugation, otherwise (with probability $1-p_{c}$ ) perform a left-insertion.

- If conjugation is selected, choose $x \in \mathcal{S}$ with uniform probability and perform a conjugation by $x$ as described above to obtain $w^{\prime \prime}$. Then $w_{n+1}$ is chosen according to the rule

$$
w_{n+1}= \begin{cases}w^{\prime \prime}, & \text { with probability } \min \left\{1, \frac{\left(\left|w^{\prime \prime}\right|+1\right)^{1+\alpha}}{(|w|+1)^{1+\alpha}} \cdot \frac{\beta^{\left|w^{\prime \prime}\right|} \mid}{\beta|w|}\right\} ; \\ w_{n}, & \text { otherwise. }\end{cases}
$$

- If left-insertion is selected, choose $R \in \mathcal{R}$ with probability $P(R)$ and a location $m \in\left\{0,1,2, \ldots,\left|w_{n}\right|\right\}$ with uniform probability. Peform a leftinsertion of $R$ at $m$ as described above to obtain $w^{\prime \prime}$. Then $w_{n+1}$ is chosen according to the rule

$$
w_{n+1}= \begin{cases}w^{\prime \prime}, & \text { with probability } \min \left\{1, \frac{\left(\left|w^{\prime \prime}\right|+1\right)^{\alpha}}{(|w|+1)^{\alpha}} \cdot \frac{\beta^{\left|w^{\prime \prime}\right|}}{\beta^{|w|}}\right\} ; \\ w_{n}, & \text { otherwise. }\end{cases}
$$

An implementation of a Markov chain which includes probabilistic rules under which moves are accepted or rejected is known as a Metropolis style algorithm. By including these specific rejection probabilities, we are able to establish the detailed balance condition, which we describe next. Notice that equations (2.4) and (2.5) are very similar except that the power of $1+\alpha$ is changed to $\alpha$. This small difference is required in order to satisfy the detailed balance condition. 
We point out to the reader that the Markov chain we have described is not a random walk on the Cayley graph of the group. Rather it executes a random walk on the set of trivial words $\mathcal{X}$. We can think of two points $x_{1}, x_{2} \in \mathcal{X}$ being connected by a weighted directed edge if the corresponding words are linked by a single conjugation or left-insertion where the weight is the appropriate probability.

2.3. The sample distribution. In this subsection we prove properties of the Markov chain defined by the transitions described above. Much of the results in this section are standard in the theory of Markov chains, but for completeness we include all relevant details. First let us define some useful notation. Define

$$
\operatorname{Pr}(u \rightarrow v)=\text { probability of tranforming } u \text { to } v \text { by an elementary move. }
$$

as per equations (2.4) and 2.5). Define

$$
\operatorname{Pr}_{n}(u \rightarrow v)=\text { probability of tranforming } u \text { to } v \text { by } n \text { elementary moves. }
$$

Definition 2.4. A Markov chain is said to be irreducible if there is a non-zero probability of moving between any two given states in a finite number of elementary moves.

A Markov chain is said to be aperiodic when for any two states $u, v$ there exists an integer $N_{0}$ so that for all $N>N_{0}$

$$
\operatorname{Pr}_{N}(u \rightarrow v)>0 \text {. }
$$

That is, if the algorithm is in state $u \in \mathcal{X}$, there is a positive probability of reaching $v \in \mathcal{X}$ in $N$ elementary moves for all $N>N_{0}$.

A Markov chain that is both irreducible and aperiodic is said to be ergodic.

Lemma 2.5. The Markov chain is irreducible.

Proof. By Lemma 2.3, there exists a sequence of moves that transforms any given state to any other given state. The probability of executing that sequence is positive, since the probability of any one move in the sequence is positive.

Lemma 2.6. The Markov chain is aperiodic.

Proof. By the Lemma 2.3. for any $u, v \in \mathcal{X}$ there is finite sequence of elementary moves that starts at $u$ and finishes at $v$. Let $N_{0}$ be the length of this sequence. Once the chain reaches this final state, $v$, there is a positive probability that any further moves leaves the algorithm in the same state. Thus the algorithm is aperiodic.

The previous two lemmas imply that the the Markov is ergodic since it is both irreducible and aperiodic.

Definition 2.7. Let $\pi$ be some probability distribution over the state space of a given Markov chain. The chain is said to satisfy the detailed balance condition with respect to $\pi$ when

$$
\pi(u) \cdot \operatorname{Pr}(u \rightarrow v)=\pi(v) \cdot \operatorname{Pr}(v \rightarrow u)
$$

for any two states $u, v$ in the chain.

Note that $\pi$ is a probability distribution over the states, while $\operatorname{Pr}(u \rightarrow v)$ is the probability of a particular transition in the Markov chain. Detailed balance describes how these probabilities interact. The main reason to consider detailed balance is that it implies that $\pi$ is the stationary distribution under the Markov chain, which we now define. 
Definition 2.8. A probability distribution $\pi$ over the states of a Markov chain is stationary if

$$
\pi(u)=\sum_{v} \operatorname{Pr}(v \rightarrow u) \pi(v)
$$

That is, $\pi$ is unchanged by a single step of the chain.

Lemma 2.9. If a Markov chain satisfies detailed balance with respect to $\pi$, then $\pi$ is stationary.

Proof. Assume that detailed balance is satisfied, then

$$
\pi(u) \cdot \operatorname{Pr}(u \rightarrow v)=\pi(v) \cdot \operatorname{Pr}(v \rightarrow u) .
$$

Summing over all states $v$ then gives

$$
\pi(u) \sum_{v} \operatorname{Pr}(u \rightarrow v)=\sum_{v} \pi(v) \operatorname{Pr}(v \rightarrow u)
$$

Since $\sum_{v} \operatorname{Pr}(u \rightarrow v)=1$ the result follows.

Lemma 2.10. Let $\pi$ be a probability distribution on $\mathcal{X}$ given by

$$
\pi(u)=\frac{(|u|+1)^{1+\alpha} \beta^{|u|}}{Z}
$$

where $Z$ is a normalising constant. The Markov chain defined above satisfies the detailed balance condition with respect to $\pi$.

We note that the normalising constant exists and is finite when $\beta$ is sufficiently small. We discuss this point further in the next section.

Proof. Let $u, v \in \mathcal{X}$. There are three possibilities: there is no single elementary move transforming $u$ to $v$ or vice-versa; $u$ and $v$ are separated by a single conjugation move; $u$ and $v$ are separated by a single left-insertion.

If there is no single elementary move between $u$ and $v$, then $\operatorname{Pr}(u \rightarrow v)=\operatorname{Pr}(v \rightarrow$ $u)=0$ and the detailed balance condition is trivially satisfied.

Now suppose that $v$ was obtained from $u$ by a conjugation as described above. Define

$$
p_{u v}=\frac{(|v|+1)^{1+\alpha}}{(|u|+1)^{1+\alpha}} \frac{\beta^{|v|}}{\beta^{|u|}} \quad p_{v u}=p_{u v}^{-1}
$$

The transition probabilities are

$$
\operatorname{Pr}(u \rightarrow v)=\frac{1}{|\mathcal{S}|} \min \left\{1, p_{u v}\right\}, \quad \operatorname{Pr}(v \rightarrow u)=\frac{1}{|\mathcal{S}|} \min \left\{1, p_{v u}\right\}
$$

The factor of $|\mathcal{S}|$ arises because we have to choose the correct conjugating element from $\mathcal{S}$. Note that $p_{u v} \leq 1$ if and only if $p_{v u} \geq 1$. So without loss of generality, assume that $p_{u v} \leq 1, p_{v u} \geq 1$. Then

$$
\operatorname{Pr}(u \rightarrow v)=\frac{p_{u v}}{|\mathcal{S}|}, \quad \quad \operatorname{Pr}(v \rightarrow u)=\frac{1}{|\mathcal{S}|} .
$$

Hence we have

$$
\operatorname{Pr}(u \rightarrow v)=p_{u v} \cdot \operatorname{Pr}(v \rightarrow u) .
$$


Next assume that $v$ is obtained from $u$ by a left-insertion of $R \in \mathcal{R}$. Let

$$
q_{u v}=\frac{(|v|+1)^{\alpha}}{(|u|+1)^{\alpha}} \frac{\beta^{|v|}}{\beta^{|u|}} \quad q_{v u}=q_{u v}^{-1}
$$

The transition probabilities are given by

$$
\operatorname{Pr}(u \rightarrow v)=\frac{P(R)}{|w|+1} \min \left\{1, q_{u v}\right\}, \quad \operatorname{Pr}(v \rightarrow u)=\frac{P\left(R^{-1}\right)}{|v|+1} \min \left\{1, q_{v u}\right\}
$$

where $P(R)$ is the probability of choosing the relation $R$ and the factor of $|u|+1$ arises from choosing the correct position to insert $R$. Recall that $P$ was chosen so that $P(R)=P\left(R^{-1}\right)$ for any $R \in \mathcal{R}$. Without loss of generality assume that $q_{u v} \leq 1$ so that $q_{v u} \geq 1$ and then

$$
\operatorname{Pr}(u \rightarrow v)=\frac{P(R)}{|u|+1} \cdot q_{u v}, \quad \operatorname{Pr}(v \rightarrow u)=\frac{P\left(R^{-1}\right)}{|v|+1}=\frac{P(R)}{|v|+1}
$$

and so

$$
\operatorname{Pr}(u \rightarrow v)=\frac{|v|+1}{|u|+1} \cdot q_{u v} \cdot \operatorname{Pr}(v \rightarrow u)=p_{u v} \cdot \operatorname{Pr}(v \rightarrow u)
$$

Notice equation (2.13) is identical to equation (2.17). This equation can be rewritten in a more symmetric form as

$$
(|u|+1)^{1+\alpha} \beta^{|u|} \operatorname{Pr}(u \rightarrow v)=(|v|+1)^{1+\alpha} \beta^{|v|} \operatorname{Pr}(v \rightarrow u) .
$$

Dividing by the normalising constant we obtain the detailed balance criterion

$$
\pi(u) \cdot \operatorname{Pr}(u \rightarrow v)=\pi(v) \cdot \operatorname{Pr}(v \rightarrow u) .
$$

As noted above, it is possible that two states $u, v$ are connected by more than one elementary move. In this case $\operatorname{Pr}(u \rightarrow v)$ is the sum of the probabilities for each elementary move, as is $\operatorname{Pr}(v \rightarrow u$ ) (by Lemmas 2.1 and 2.2), so detailed balance is preserved.

The next result shows that detailed balance implies uniqueness of the stationary distribution. Though it is a standard result in the theory of Markov chains, again we include it here for completeness.

Lemma 2.11. The distribution $\pi$ described in the previous lemma is the unique distribution on $\mathcal{X}$ for which the algorithm satisfies detailed balance.

Proof. Suppose there is another distribution $\varphi$ on $\mathcal{X}$ for which detailed balance is satisfied. If $\varphi \neq \pi$ then there exists a state $y \in \mathcal{X}$ so that $\varphi(y)>\pi(y)$.

So for every state $y^{\prime}$ that is connected to $y$ by an elementary move $(i . e$. for which $\left.\operatorname{Pr}\left(y \rightarrow y^{\prime}\right)>0\right)$ we have

$$
\varphi\left(y^{\prime}\right) \operatorname{Pr}\left(y^{\prime} \rightarrow y\right)=\varphi(y) \operatorname{Pr}\left(y \rightarrow y^{\prime}\right)>\pi(y) \operatorname{Pr}\left(y \rightarrow y^{\prime}\right)=\pi\left(y^{\prime}\right) \operatorname{Pr}\left(y^{\prime} \rightarrow y\right)
$$

Hence $\varphi\left(y^{\prime}\right)>\pi\left(y^{\prime}\right)$. Thus $\varphi(x)>\pi(x)$ for all $x$ reachable from $y$. Since the chain is irreducible, $\varphi(x)>\pi(x)$ for all $x \in \mathcal{X}$. This contradicts the assumption that $\varphi$ is a probability distribution.

Now that we have established the above properties of the Markov chain, we can make use of the Fundamental Theorem of Markov chains: 
Theorem 2.12 (Fundamental Theorem of Markov chains). If a Markov chain $\mathcal{M}$ is irreducible and aperiodic then it has a unique stationary distribution $\varphi$. Moreover,

$$
\operatorname{Pr}_{n}(x \rightarrow y) \rightarrow \phi(y) \quad \text { as } n \rightarrow \infty
$$

for all $x, y$ in the state space of $\mathcal{M}$.

The above theorem can be found in most standard texts on stochastic processes - see, for example, 21, 29, 34.

Corollary 2.13. Given any two states $u, v \in \mathcal{X}$

$$
\operatorname{Pr}_{n}(u \rightarrow v) \rightarrow \pi(v) \quad \text { as } n \rightarrow \infty
$$

where $\pi(u)$ is the unique stationary distribution of the Markov chain

$$
\pi(u)=\frac{(|u|+1)^{1+\alpha} \beta^{|u|}}{Z}
$$

where $Z$ is a normalising constant which depends on $\alpha, \beta, p_{c}$ and the group presentation.

Proof. By the previous lemmas, our Markov chain satisfies the conditions of the theorem. Further, since $\pi$ is a stationary distribution for our Markov chain, it must, by the same theorem, be the unique stationary distribution.

The above corollary implies that we can use our Markov chain to sample trivial words from a given finitely presented group with a specific distribution, $\pi$. When $\alpha=-1, \pi$ is the Boltzmann or Gibbs distribution. For other values of $\alpha$ we can think of $\pi$ as a "stretched" Boltzmann distribution. Also note that $\pi$ does not depend of the details of the word, but only on its length. So if two words have the same length then they are sampled with the same probability.

In the next section we examine the mean length of sampled words and describe how this can inform us about the cogrowth of the group.

2.4. Mean length of sampled words. As demonstrated in the previous section, the Markov chain converges to a stretched Boltzmann distribution, $\pi$. We defined $\pi$ above in terms of a normalising constant, $Z$, which we now make more precise. Since we require $\sum_{w \in \mathcal{X}} \pi(w)=1$, we must have

$$
Z=\sum_{w \in \mathcal{X}}(|w|+1)^{1+\alpha} \beta^{|w|}
$$

which can be written in terms of the cogrowth function

$$
Z=\sum_{n \geq 0} c(n) \cdot(n+1)^{1+\alpha} \beta^{n} .
$$

This sum converges to a finite value for $0 \leq \beta<\beta_{c}$, where $\beta_{c}$ is

$$
\beta_{c}=\limsup _{n \rightarrow \infty} c(n)^{-1 / n} .
$$

and is independent of the parameters $\alpha, p_{c}$. Note that, $\beta_{c}$ is exactly the radius of convergence of cogrowth series

$$
C(z)=\sum_{n \geq 0} c(n) z^{n} .
$$


This demonstrates the link between the behaviour of the Markov chain and the cogrowth of the underlying group.

Let us now turn to expected length of words sampled by the Markov chain. Under the stationary distribution, $\pi$, the expected length of words in $\mathcal{X}$ is given by

$$
\begin{aligned}
\mathbb{E}(|w|) & =\sum_{w \in \mathcal{X}}|w| \pi(w)=\sum_{w}|w| \frac{(|w|+1)^{1+\alpha} \beta^{|w|}}{Z} . \\
& =\frac{\sum_{n \geq 0} n(n+1)^{1+\alpha} c(n) \beta^{n}}{Z} .
\end{aligned}
$$

With the Markov chain as described we can select a particular value of $\beta$ and compare samples from the chain to exact results for groups where the cogrowth series is known (such as $\mathbb{Z}^{2}$ ). In practice we would like to examine how the expected length changes with $\beta$. When $\beta$ is very small, $\mathbb{E}(|w|) \equiv\langle n\rangle$ should be small since shorter words are favoured. As $\beta$ grows the expectation will increase. When $\beta$ exceeds $\beta_{c}$ the expectation will cease to converge and words sampled by the chain will become longer and longer.

Rather than running many independent copies of the chain at distinct $\beta$-values we use a technique known as Multiple Markov chains or parallel tempering which samples at a set of distinct $\beta$-values simultaneously. We refer the reader to [17, 35] for a detailed description of this method.

When $\alpha=-1$ we can write the mean length explicitly as the log-derivative of $C(z)$ :

$$
\mathbb{E}(|w|)=\left.\left(\frac{z C^{\prime}(z)}{C(z)}\right)\right|_{z=\beta}=\left.\left(z \frac{\mathrm{d}}{\mathrm{d} z} \log C(z)\right)\right|_{z=\beta} .
$$

One can do similarly for $\alpha=0,1,2, \ldots$

$$
\mathbb{E}(|w|)=\left.(\underbrace{\frac{\mathrm{d}}{\mathrm{d} z} z \frac{\mathrm{d}}{\mathrm{d} z} z \cdots}_{1+\alpha \text { times }}\left(z \frac{\mathrm{d}}{\mathrm{d} z} \log C(z)\right))\right|_{z=\beta} .
$$

We will make use of this expression in Section 3 for groups where the cogrowth series is known exactly. This will allow us to compare numerical results from an implementation of the Markov chain against exact results. Note that in the graphs that follow below we will use $\langle n\rangle$ to denote mean length in place of $\mathbb{E}(|w|)$.

2.5. Alternate sets of elementary moves. While we have implemented the above Markov chain using conjugations and left-insertions as elementary moves, other moves are possible. The proof of Lemma 2.3 relies on conjugations but only a subset of left-insertions. In particular, it only requires left-insertions in $w$ at position $m=0$, that is, appending a relation to the extreme right of $w$.

Hence Corollary 2.13 would still hold for a Markov chain using the following elementary moves

- conjugation by $x \in \mathcal{S}$ : given $w, w^{\prime}=x^{-1} w x$, and

- append $R \in \mathcal{R}$ : given $w, w^{\prime}=w \mapsto w R$.

Note that appending $R$ is always reversible by appending $R^{-1}$.

Since every word in the state space of the chain represents the identity element of the group, we could also introduce a rotation move 
- rotate at $k$ : given $w=u v$ with $|u|=k, w^{\prime}=v u$.

In order to ensure this move is reversible by another rotation one needs to ensure that no cancellations occur upon freely reducing $w^{\prime}$. With this restriction a rotation by $k$ can always be reversed by a rotation at $|w|-k$.

Of course, if the set of elementary moves is changed then the transition probabilities described by equations 2.13 and (2.17) need to be updated in order to satisfy detailed balance.

2.6. Avoiding the empty word. The Markov chain can be implemented to sample from the state space of non-empty trivial words. ie from $\mathcal{X}^{\prime}=\mathcal{X}-\{\epsilon\}$. To do this we alter equations (2.13) and (2.17) so that if $w^{\prime \prime}=\epsilon$ then $w_{n+1}=w_{n}$. That is, if an elementary move attempts to step to the empty word then it is rejected and the current word is kept.

The following lemma shows that with this restriction the Markov chain remains irreducible.

Lemma 2.14. The Markov chain described above with elementary moves altered to avoid the empty word is irreducible on $\mathcal{X}^{\prime}$, except when applied to the presentation $\left\langle a \mid a^{k}\right\rangle, k \in \mathbb{N}$.

Proof. Let $w$ be a word in $\mathcal{X}^{\prime}$. By Lemma 2.3 it can be reduced to the empty word by a sequence of elementary words. The penultimate word in this sequence must be a relator; denote it $r_{w}$.

Hence if $u, v \in \mathcal{X}^{\prime}$ they can be reduced by sequences of elementary moves to relators $r_{u}, r_{v}$. There are three possibilities

- If $r_{u}=r_{v}$ then reversing one of the sequences of moves shows that $u, v$ are connected a sequence of elementary moves.

- If $r_{u} \neq r_{v}, r_{v}^{-1}$ then write $r_{u}=r_{1} r_{2}$ and $r_{v}=r_{2}^{-1} r_{3}$, where $r_{2}$ is as large as possible. Note that $r_{2}$ could be the empty word. Now

- left-insert $r_{v}$ after $r_{u}$ to obtain $r_{u} r_{v}=r_{1} r_{2} r_{2}^{-1} r_{3} \mapsto r_{1} r_{3} \neq \epsilon$ (after free reduction).

- left-insert $r_{1}^{-1} r_{2}^{-1}$ after $r_{1}$ to obtain $r_{1} r_{1}^{-1} r_{2}^{-1} r_{3} \mapsto r_{2}^{-1} r_{3}=r_{v}$.

Note that since $r_{u}=r_{1} r_{2} \in \mathcal{R}$ so is $r_{1}^{-1} r_{2}^{-1}$ since it is a cyclic rotation of $r_{u}^{-1}=r_{2}^{-1} r_{1}^{-1}$. Thus there must be a sequence of elementary moves connecting $u$ and $v$.

- If $r_{u}=r_{v}^{-1}$ then find another relator $r_{w} \in \mathcal{R}$ so that $r_{w} \neq r_{u}, r_{u}^{-1}$ (we discuss the existence of $r_{w}$ at the end of the proof). Now use the previous case to transform $r_{u} \mapsto r_{w}$ and again to transform $r_{w} \mapsto r_{v}$. This creates a sequence of elementary moves connecting $u$ and $v$.

In all three cases there is a sequence of moves connecting $u$ and $v$. Since the probability of each move in the sequence is positive, so the probability of the sequence is positive.

Note that the last case breaks down if we are unable to find $r_{w} \neq r_{u}, r_{u}^{-1}$. If the presentation has two or more relations, then simply pick $r_{w}$ to be a cyclic permutation of a relation that is not $r_{u}$. If the group has a single relation, then let $r_{w}$ be a cyclic permutation of $r_{u}$ different from $r_{u}, r_{u}^{-1}$. If no such $r_{w}$ exists then all cyclic permutations of $r_{u}, r_{u}^{-1}$ must be equal to either $r_{u}$ or $r_{u}^{-1}$. We now show that this implies the single relation must be of the form $a^{k}$.

Let $r_{u}=x_{1} x_{2} \ldots x_{k}$ be a word in $\mathcal{S}^{*}$ and let $w=x_{2} \ldots x_{k} x_{1}$ be a cyclic rotation of $r_{u}$. If $w=r_{u}$ then we have $x_{2}=x_{1}, x_{3}=x_{2}, \ldots, x_{k}=x_{1}$ and thus all the 
symbols in $r_{u}$ must be the same. On the other hand, if $w=r_{u}^{-1}=x_{k}^{-1} \ldots x_{1}^{-1}$ then we must have that $x_{1}=x_{1}^{-1}$ which is a contradiction. So we must have that $r_{u}=x^{k}$ for some $x \in \mathcal{S}$.

Now if the group has two or more generators then we can proceed as follows:

- Without loss of generality, write $r_{u}=a^{k}$. Conjugate by another generator $b$ (again without loss of generality) to obtain $b^{-1} a^{k} b$. Left-insert $a^{-k}$ at the end of the word, giving $b^{-1} a^{k} b a^{-k}$. Rotate the word by a sequence of conjugations to $b a^{-k} b^{-1} a^{k}$. Left-insert $a^{-k}$ at the end of the word giving $b a^{-k} b^{-1}$. Finally conjugate by $b$ to arrive at $a^{-k}=r_{u}^{-1}=r_{v}$.

Thus one can connect $r_{u}$ and $r_{v}$, and so $u$ and $v$, by a sequence of elementary moves. Again, since each move in the sequence has positive probability, so does the whole sequence.

Finally if the group has only a single generator then it must be of the form $\left\langle a \mid a^{k}\right\rangle$ for some $k \in \mathbb{N}$. Write $w=a^{n k}$ for some $n \in \mathbb{N}$. Conjugating $w$ leaves it unchanged, while a left-insertion maps $w \mapsto a^{(n-1) k}, a^{n k}, a^{(n+1) k}$. Hence it is not possible to transform $a^{k}$ to $a^{-k}$ by a sequence of elementary moves without passing through the empty word $a^{0}$.

Notice that the proofs of Lemmas 2.6 and 2.10 remain unchanged. Hence Corollary 2.13 holds and the Markov chain on $\mathcal{X}^{\prime}$ converges to the same stationary distribution. The only difference is that the normalising constant, $Z$, changes; it is reduced by exactly 1 .

When we implemented the Markov chain on $\mathcal{X}$ as described in subsections 2.1 and 2.2 , we found that it would spend a very large time sampling the empty word. It must do this since the empty word is highly probable under the limit distribution. In order to force the chain to sample longer words we implemented the chain on $\mathcal{X}^{\prime}$ and used it to generate the results discussed in Section3. Note that when computing the exact expected mean length of the chain on $\mathcal{X}^{\prime}$ using equation 2.28, we must ensure that $C(z)$ does not count the empty word and so we replace $C(z)$ by $C(z)-1$.

2.7. Infinitely related groups. Another possible extension of the algorithm is to consider groups which have a finite number of generators but an infinite number of relations, for example

$$
\left.\mathbb{Z} \imath \mathbb{Z}=\langle a, b|\left[a^{b^{i}}, a^{b^{j}}\right] \text { with } i, j \in \mathbb{Z}\right\rangle .
$$

When performing a left-insertion we choose a particular relation $R \in \mathcal{R}$ with probability $P(R)$. The only restrictions on this distribution $P$, are that $P(R)>0$ for all $R \in \mathcal{R}$ and that $P(R)=P\left(R^{-1}\right)$. As long as these conditions are satisfied, then the detailed balance condition will be satisfied and Corollary 2.13 will hold. Consequently there is no requirement in the above analysis that $\mathcal{R}$ be finite.

We implemented our chain on the above presentation of $\mathbb{Z} \imath \mathbb{Z}$ with different choices of $P$. The statistics collected from those Markov chains did appear to be independent of the distribution $P$, as one would hope, and it was also consistent with the amenability of the group. We have not included this extension in the present work; we plan to include it in a future work on precise analysis of statistics collected from the chain, together with other infinitely presented groups. 


\section{NUMERICAL RESULTS}

In this section we discuss the application of the Markov chain to concrete examples of finitely presented groups. We chose a range of amenable and non-amenable groups including those for which the cogrowth series is known exactly. Additionally we have applied the Markov chain to Thompson's group $F$ - whose amenability is currently an open problem.

The chain on $\mathcal{X}^{\prime}$ was implemented in c++ with words stored as linked lists. The linked-list data structure makes the computer code associated with conjugation and left-insertion relatively straight-forward. To ensure correctness of the implementation, two separate programs were created independently by the second and third authors, and results compared. We used the GNU Scientific Library ${ }^{1}$ to generate pseudo-random numbers to decide transitions within the chain. At each beta value we sampled approximately $10^{10}$ elementary moves. Each run consisted of $100 \beta$ values and took approximately 1 week on a single node of a computing cluster at the Western Canada Research Grid (Westgrid). Each node was roughly equivalent to a modest desktop computer running Linux.

We remark that for some groups it is easier to compute the generating function of the number of all words equivalent to the identity, not just those that are freely reduced. This series for $\mathbb{Z}^{2}$, for example, is

$$
D(z)=\sum_{n \geq 0}\left(\begin{array}{c}
2 n \\
n
\end{array}\right)^{2} z^{2 n}=\frac{2}{\pi} K(4 z)
$$

where $K(z)$ is the complete elliptic integral of the first kind. We refer the reader to [15] for a short proof of the above. It is then straight-forward to transform this series to cogrowth series using the following result of Woess:

Lemma 3.1 (Lemma 1 of [37]). Let $d(n)$ be the number of words of length $n$ equal to the identity in a given group presentation, and let $D(z)=\sum d(n) z^{n}$ be the associated generating function. Let $2 q=|\mathcal{S}|$, then

$$
C(z)=\frac{1-q+q \sqrt{1-4(2 q-1) z^{2}}}{1-4 q^{2} z^{2}} D\left(\frac{1-\sqrt{1-4(2 q-1) z^{2}}}{2(2 q-1) z}\right)
$$

We remind the reader that we have implemented the chain on $\mathcal{X}^{\prime}=\mathcal{X}-\{\epsilon\}$ and so we must replace $C(z)$ by $C(z)-1$ when computing exact expectations using equation 2.28.

3.1. Application to $\mathbb{Z}^{2}$. Our first example is $\mathbb{Z}^{2}$ which is amenable and whose cogrowth series is known exactly (see above). In Figure 1 we plot the exact expectation of the length of words as a function of $\beta$ with $\alpha=1$ (the solid curve in the plot); this curve was computed by combining equations $(3.2),(3.1)$ and $(2.28)$.

We overlay the average length of words observed in the implementation of our chain running with $\alpha=1$ and a range of $\beta$-values (the crosses in the plot). The figure demonstrates that there is excellent agreement between the exact results and the numerical estimates. Similar agreement was found for different values of $\alpha$.

Notice that the data show that the mean length increases with $\beta$ and that it becomes larger and larger as $\beta$ approaches $1 / 3$. Indeed, since the group is amenable,

\footnotetext{
${ }^{1}$ Available at http://www.gnu.org/software/gsl/ at time of writing.
} 


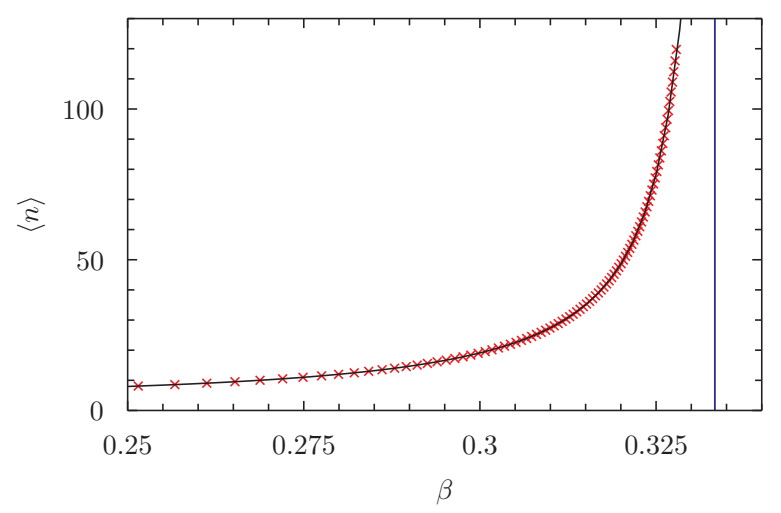

Figure 1. The mean length of sampled words plotted against $\beta$ for the standard presentation of $\mathbb{Z}^{2}$ with $\alpha=1$. The crosses indicate data obtained from an implementation of the algorithm while the curve indicates the expectation derived from the exact cogrowth series for the group. The vertical line indicates $\beta_{c}=1 / 3$.

we know that the cogrowth is exactly 3 (by [18, 7 ), and so the radius of convergence of the cogrowth series is $1 / 3$.

3.2. Application to examples of Kouksov. The cogrowth series is known in closed form for very few groups. In [23] Kouksov gives explicit formulae for some free products. We examined the following three:

$$
\begin{aligned}
& K_{1}=\left\langle a, b \mid a^{2}, b^{3}\right\rangle, \\
& K_{2}=\left\langle a, b \mid a^{3}, b^{3}\right\rangle, \\
& K_{3}=\left\langle a, b, c \mid a^{2}, b^{2}, c^{2}\right\rangle .
\end{aligned}
$$

whose cogrowth series are given by

$$
\begin{aligned}
& C_{1}(t)=\frac{(1+t)\left(f_{1}(t)+\left(2-t+6 t^{2}\right) \sqrt{f_{2}(t)}\right)}{2(1-3 t)\left(1+3 t^{2}\right)\left(1+3 t+3 t^{2}\right)\left(1-t+3 t^{2}\right)} \\
& C_{2}(t)=\frac{(1+t)\left(-t+\sqrt{1-2 t-t^{2}-6 t^{3}+9 t^{4}}\right)}{(1-3 t)\left(1+2 t+3 t^{2}\right)}, \text { and } \\
& C_{3}(t)=\frac{-1-5 t^{2}+3 \sqrt{1-22 t^{2}+25 t^{4}}}{2\left(1-25 t^{2}\right)}
\end{aligned}
$$

where $f_{1}(t)=-t+t^{2}-8 t^{3}+3 t^{4}-9 t^{5}$ and $f_{2}(t)=1-2 t+t^{2}-6 t^{3}-8 t^{4}-$ $18 t^{5}+9 t^{6}-54 t^{7}+81 t^{8}$. The radii of convergence of these cogrowth series are $0.3418821478,0.3664068598$ and 0.2192752634 respectively (to ten significant digits). Hence the cogrowth is strictly smaller than the value required for amenability being 3,3 and 5 , respectively. Indeed each of these contains a non-abelian free subgroup and so are non-amenable; in the case of the groups $K_{1}$ and $K_{2}$ the free subgroups are $F\left((a b),\left(a b^{-1}\right)\right)$, and for $K_{3}$ the free subgroup is $F((a b),(a c))$.

In Figure 2 we compare data obtained from our algorithm with the exact expectation, which was computed by combining the exact cogrowth series above with equation 2.28). Note that because the chain avoids the empty word, we modify 


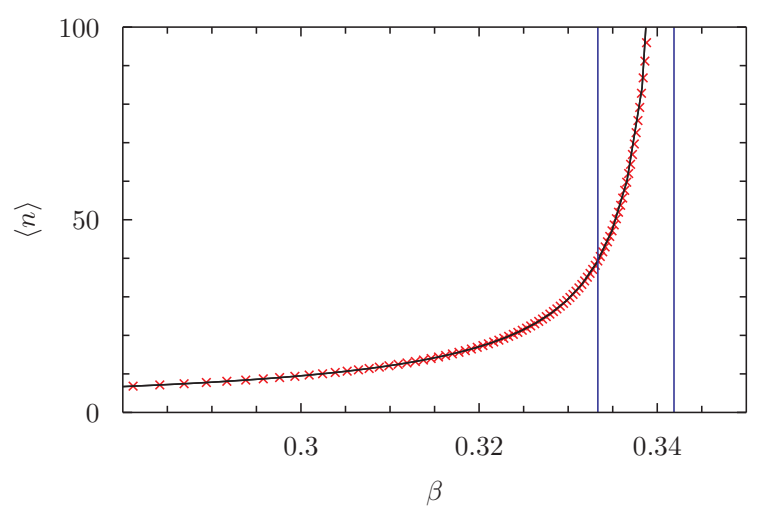

(A) $\left\langle a, b \mid a^{2}, b^{3}\right\rangle$ sampled with $\alpha=0$.

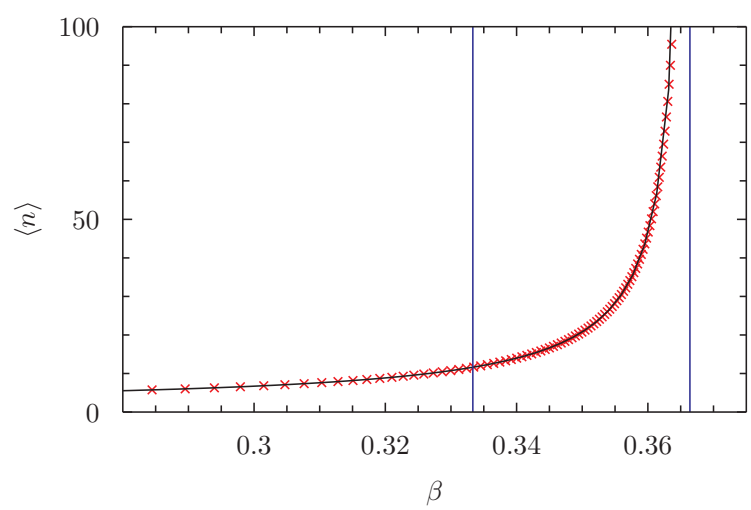

(в) $\left\langle a, b \mid a^{3}, b^{3}\right\rangle$ sampled with $\alpha=0$.

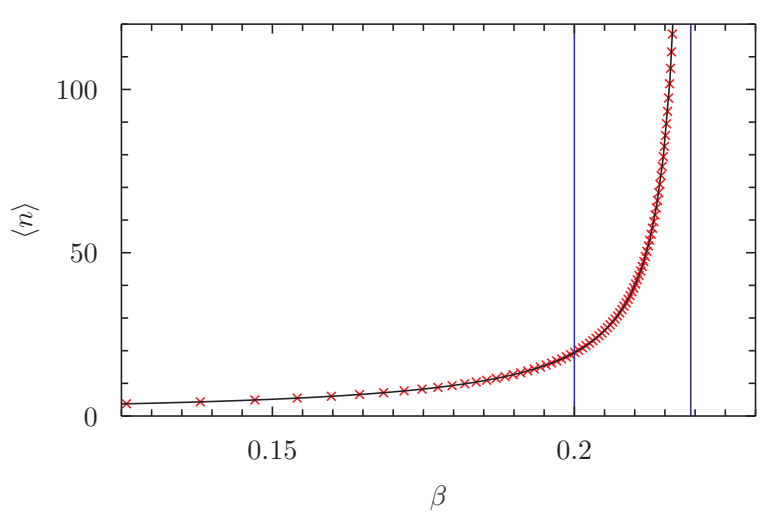

(c) $\left\langle a, b, c \mid a^{2}, b^{2}, c^{2}\right\rangle$ sampled with $\alpha=1$.

Figure 2. Mean length of sampled words plotted against $\beta$ for $K_{1}, K_{2}$ and $K_{3}$. The crosses indicate data obtained from the algorithm, while the curves indicate the expectation derived from the exact cogrowth series for each group. The first vertical lines in each plot indicates $\beta=1 / 3,1 / 3,1 / 5$ (respectively) and also the reciprocal of the cogrowth where the statistic will diverge - being $0.3418821478,0.3664068598$ and 0.2192752634 respectively. 
the above generating functions by subtracting 1 from each (being the contribution from the empty word). As was the case for $\mathbb{Z}^{2}$, there is excellent agreement between the numerical and exact results.

3.3. Application to $\operatorname{BS}(N, N)$. The cogrowth series for $\operatorname{BS}(N, N)$ is not known in closed form for $N \geq 2$. In recent work 15 the authors and Tom Wong demonstrate that the cogrowth series for $\operatorname{BS}(N, N)=\left\langle a, b \mid a^{N} b a^{-N} b^{-1}\right\rangle$ is D-finite, that is, the series $C(z)$ satisfies a linear differential equation with polynomial coefficients. This work allows the cogrowth to be computed exactly for moderate values of $N$ in polynomial time.

It follows that the cogrowth series can be computed to (essentially) any desired number of terms. Using that truncated series and equation $(2.28)$ we the compute expectation of the length to any desired precision. In Figure 3 we display the expected mean length against data obtained from the Markov chain. As with previous examples, we see excellent agreement.

3.4. Application to $\operatorname{BS}(N, M)$ with $N \neq M$. The work [15] is mostly concerned with $\operatorname{BS}(N, N)$, but the central enumerative result (Proposition 3.6 in [15]) also holds for $\operatorname{BS}(N, M)=\left\langle a, b \mid a^{N} b a^{-M} b^{-1}\right\rangle$. The authors derive a system of three $q$-algebraic equations which can be iterated to compute the first few terms of $C(z)$. This is more efficient than a brute-force approach but it still requires exponential time and memory.

Explicitly the authors define a two-variable generating function

$$
G(z ; q)=\sum_{n} z^{n} g_{n}(q) \quad \text { where } g_{n}(q)=\sum_{k} g_{n, k} q^{k}
$$

where $g_{n, k}$ is the total number of words (not just those that are freely reduced) of length $n$ equal to $a^{k}$. Thus $g_{n, 0}=d(n)$ defined in Lemma 3.1. When $N=M, g_{n}(q)$ has at most $2 n+1$ non-zero terms, however when $N \neq M$ the number of non-zero terms is exponential in $n$.

Due to the exponential constraint, we are only able to compute the first few terms of cogrowth series exactly. For example we were only able to compute the first 60 terms of $D(z)$ (and hence $C(z)$ by equation $(3.2)$ ) for $\mathrm{BS}(1,2)$. Using those truncated series and equation 2.28 we get a lower bound on the exact expected mean length - this is the solid curve in Figures 4 and 5

When we generated series by the above method we noticed that the polynomials $g_{n}(q)$ are dominated by the central few terms around $q^{0}$, while the other terms (being the vast majority) were negligible. This suggests an alternate means to estimate $D(z)$ (and so $C(z)$ ) - at each iteration of the system of $q$-algebraic equations we discarded all but the central $2 n+1$ terms of $g_{n, k}$. The resulting series $\tilde{G}(z ; q)$ is dominated term-by-term by the true $G(z ; q)$, but can be computed to far more terms (indeed it is comparable in effort to the computation for $\operatorname{BS}(N, N)$ described above). We have also estimated the exact expectation using this method; it also gives underestimates of the true expectation. The curve is plotted as dotted lines in Figures 4 and 5 .

In all four plots we see good agreement between the two estimates and the data from the Markov chain. As $\beta$ is increased the two estimates fall below the Markov chain data, with the estimate from truncated series distinctly lower than the estimate from approximate series. This is consistent with the Markov chain giving accurate estimates of the true expected length for $\beta$ even quite close to $\beta_{c}$. 


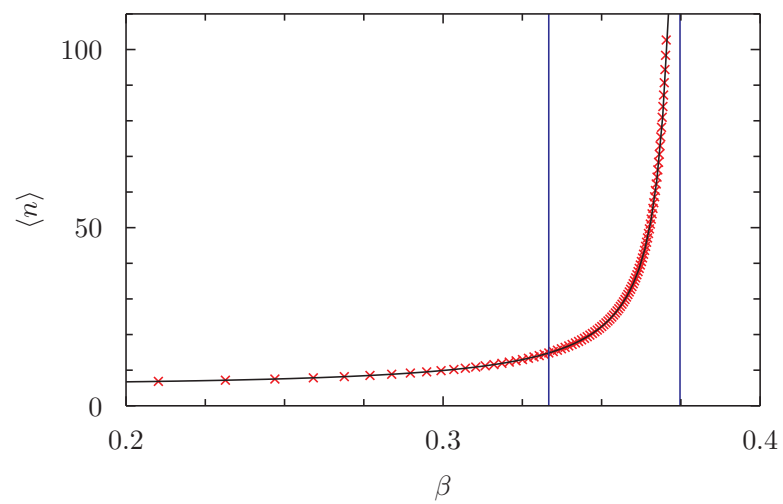

(A) $\operatorname{BS}(2,2)$ sampled with $\alpha=1$.

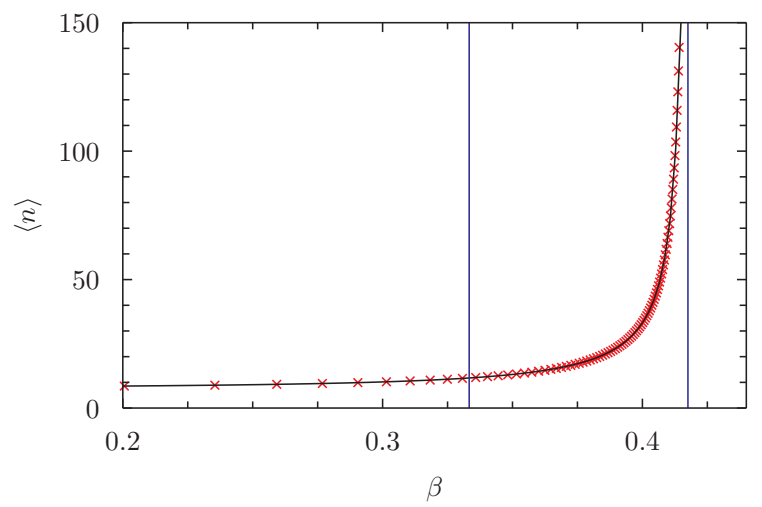

(в) $\mathrm{BS}(3,3)$ sampled with $\alpha=1$.

FiguRE 3. Mean length of sampled words plotted against $\beta$ for $\mathrm{BS}(2,2)$ and $\mathrm{BS}(3,3)$. The crosses indicate data obtained from the algorithm, while the curves indicates the expectation derived from the cogrowth series for each group. The vertical lines indicate $\beta=1 / 3$ and also the reciprocal of the cogrowth being 0.3747331572 and 0.417525628 respectively (see [15]). We see excellent agreement between our numerical data and the exact results.

In the cases of $\operatorname{BS}(1,2)$ and $\mathrm{BS}(1,3)$ we know the reciprocal of the cogrowth is $1 / 3$ since they are amenable, and the Markov chain data confers with this.

3.5. Application to the basilica group. We now turn to the first of two groups for which we know very little about the cogrowth series - namely the basilica group first studied by Grigorchuk and Zuk [19]. This group has an infinite presentation

$$
\left.G=\langle a, b|\left[a^{n},\left[a^{n}, b^{n}\right]\right] \text { and }\left[b^{n},\left[b^{n}, a^{2 n}\right]\right] \text { where } n \text { is a power of } 2\right\rangle
$$

where we have used the notation $[x, y]=x^{-1} y^{-1} x y$ and $x^{y}=y^{-1} x y$. This group embeds in the finitely presented group [19]

$$
\widetilde{G}=\left\langle a, b \mid a^{b^{2}}=a^{2},\left[\left[\left[a, b^{-1}\right], a\right], a\right]=1\right\rangle .
$$




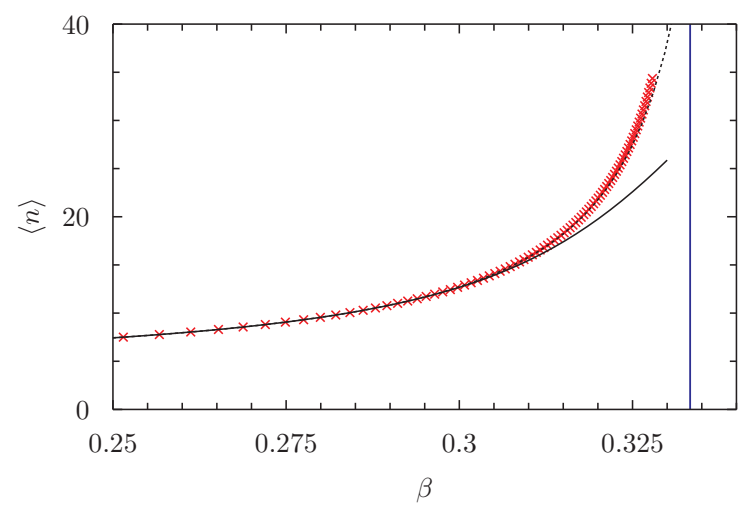

(A) $\operatorname{BS}(1,2)$ sampled with $\alpha=1$.

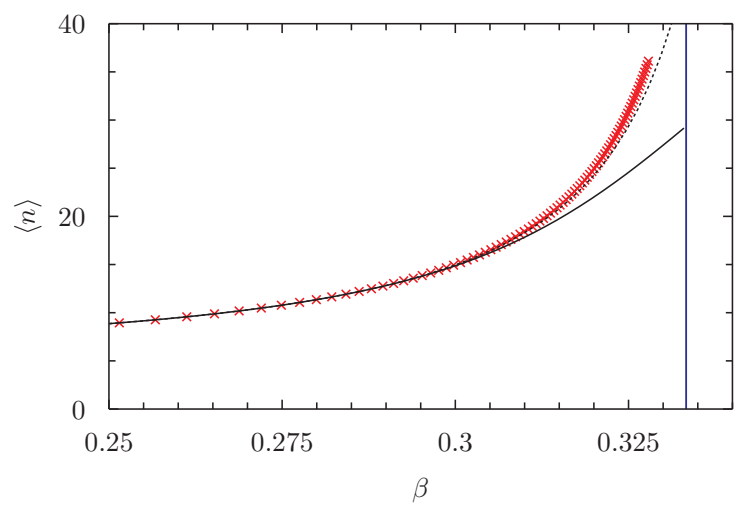

(в) $\operatorname{BS}(1,3)$ sampled with $\alpha=2$.

Figure 4. Mean length of freely reduced trivial words in Baumslag-Solitar groups $\mathrm{BS}(1,2)$ and $\mathrm{BS}(1,3)$ at different values of $\beta$ and $\alpha$ as indicated. The sampled points are indicated with crosses, while the vertical line indicates $\beta_{c}=1 / 3$. The solid line indicates estimates of the exact expectation derived from the exact but truncated cogrowth series. The dotted line indicates estimates of the expectation derived using the approximation of the cogrowth (as described in the main text). At low and moderate values of $\beta$ there is excellent agreement, but as $\beta$ increases the Markov chain lies above both of the approximations of the expectation which is consistent with the approximations being underestimates.

Bartholdi and Virag proved that both $G$ and $\widetilde{G}$ are amenable [3], and separate the classes of amenable and subexponentially amenable groups.

As noted in subsection 2.7 our algorithm can be extended to infinite presentations, however for this article we restricted our study to the finitely presented group $\widetilde{G}$. We ran the algorithm on three presentations derived from the above presentation by simple Tietze transformations (see [25] p. 89). The first is obtained from the above by putting $c=\left[a, b^{-1}\right]$, and the second by putting $c=a^{b}$. Simplification 


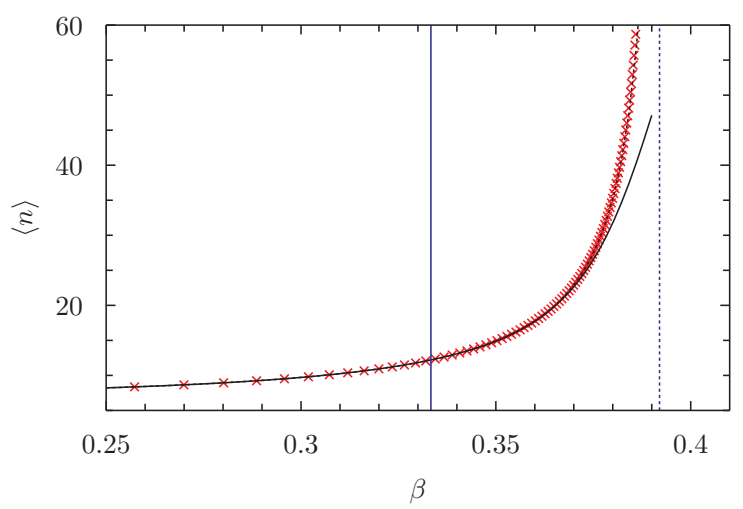

(A) $\operatorname{BS}(2,3)$ sampled with $\alpha=1$.

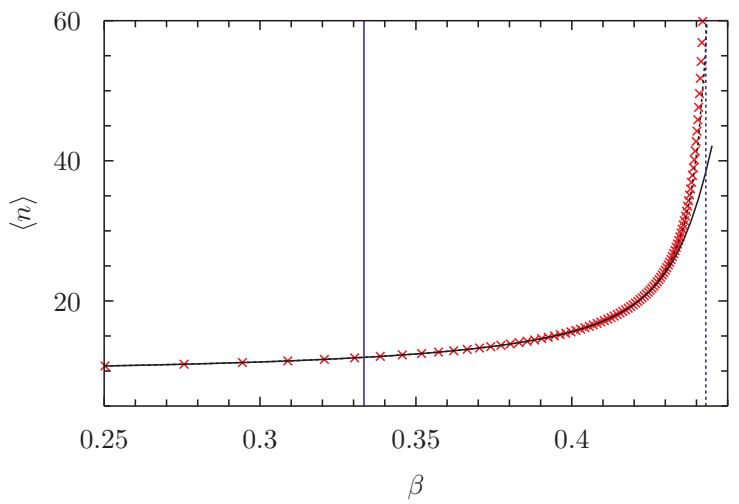

(В) $\operatorname{BS}(3,5)$ sampled with $\alpha=0$.

Figure 5. The mean length of trivial words in $\operatorname{BS}(2,3)$ and $\mathrm{BS}(3,5)$ at different values of $\beta$. The sampled points are indicated with crosses, while the solid vertical line indicates $\beta_{c}=1 / 3$. The dotted vertical lines indicate the estimated critical value of $\beta$ from analysis of the truncated series. As per the previous figure, the solid line indicates estimates of the expectation from truncated series while the dotted line indicates estimates from the approximate series (see the main text). At low and moderate values of $\beta$ there is excellent agreement, but as $\beta$ increases the Markov chain lies above both of the approximations of the expectation.

gives the representations

$$
\begin{aligned}
& \widetilde{G}=\left\langle a, b, c \mid c=\left[a, b^{-1}\right], a^{b^{2}}=a^{2},[[c, a], a]=1\right\rangle, \\
& \widetilde{G}=\left\langle a, b, c \mid c=a^{b}, c^{b}=a^{2}, c^{-1} a c a^{-1} c^{-1} a^{-1} c a=1\right\rangle .
\end{aligned}
$$

We implemented the Markov chain for both of these presentations. We plot the mean length of words sampled from the chains in Figure 6. An immediate observation is that the mean length is remarkably insensitive to changes in $\beta$. Because of this we found that our data was far harder to analyse than for the other groups 
discussed above. This is compounded by the absense of cogrowth series data for comparison.

Because this data appeared so insensitive to $\beta$, we also examined a measure of the statistical error in our estimates. To do this we consider samples from the Markov chain as a time series of length $N$. We slice this sequence into $M$ nonoverlapping blocks of length $N / M$. Let the mean length observed in the $i^{\text {th }}$ such block be denoted $\langle n\rangle_{i}$. The variance in these mean lengths and our error estimate are then given by

$$
\begin{aligned}
\operatorname{var} & =\frac{1}{M^{2}} \cdot \sum_{i}\langle n\rangle_{i}^{2}-\left(\frac{1}{M} \sum_{i}\langle n\rangle_{i}\right)^{2} \\
\operatorname{err} & =\sqrt{\frac{\operatorname{var}}{M-1}}
\end{aligned}
$$

Our typical runs consisted of around $10^{3}$ blocks each of length approximately $10^{7}$. We made estimates of autocorrelations at the highest values of $\beta$ and found them to be much shorter than the block length. This validates the above estimate of the error.

We repeated this analysis on the examples studied above (the Baumslag-Solitar groups and the examples of Kouksov), and found that the error estimates were very small. Indeed, if we were to place error-bars on our plots of the mean length they would be smaller than the crosses used to denote the data - except very close to $\beta_{c}$. This is consistent with our observation that our Markov chain data agrees closely with exact results. It also indicates another method of detecting the location of a singularity — we expect that the error estimate will diverge as $\beta \rightarrow \beta_{c}$.

We have plotted the reciprocal of our error estimate against $\beta$ for these two presentations in Figure 6. We see a much clearer signal of divergence closer to $\beta_{c}=1 / 5$ than we do for the mean length data.

We studied a third presentation, in which the relators are of shorter and comparable lengths. We set $c=a^{b}, d=\left[a, b^{-1}\right], e=[d, a]$ in equation 3.9 to obtain the presentation:

$$
\widetilde{G}=\left\langle a, b, c, d, e \mid c=a^{b}, d=\left[a, b^{-1}\right], e=[d, a], c^{b}=a^{2},[e, a]=1\right\rangle .
$$

We found that the mean-length data from this presentation was much better behaved and gave a clearer signal of a singularity at $\beta=1 / 9$. See Figure 7. We also analysed the error data and estimate that the reciprocal of the error goes to zero as $\beta \rightarrow 1.115 \pm 0.005$. The data from this presentation is consistent with the amenability of $\widetilde{G}$. Overall, the data from all three presentations is consistent with the group being amenable.

3.6. Application to the Thompson's group $F$. We now turn to our last application, Thompson's group $F$. We started by examining its standard finite presentation

$$
F=\left\langle a, b \mid\left[a b^{-1}, a^{-1} b a\right],\left[a b^{-1}, a^{-2} b a^{2}\right]\right\rangle .
$$




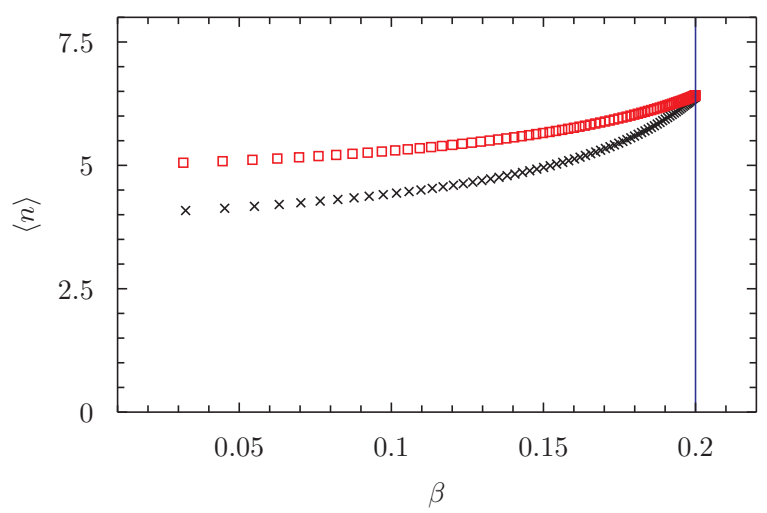

(A) Mean length with $\alpha=5$.

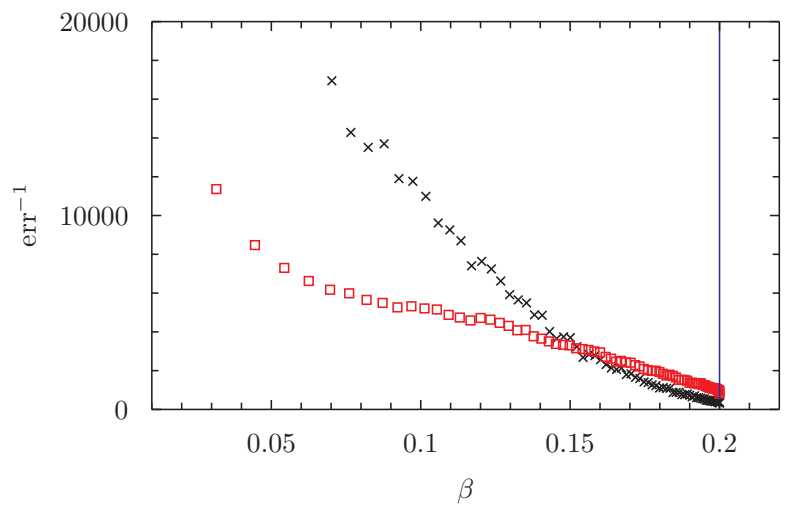

(в) $\operatorname{err}^{-1}$ with $\alpha=5$.

Figure 6. (A) The mean length of words plotted against $\beta$ for two presentations of group $\widetilde{G}$. Data points indicated by $\square, \times$ corresponds to 3.10 and (3.11) respectively. (B) The reciprocal of the estimated error against $\beta$. Notice that as $\beta \rightarrow 1 / 5$ the error begins to diverge.

In addition to this presentation, we implemented the chain on two further presentations derived using simple Tietze transformations:

$$
\begin{gathered}
F=\left\langle a, b, c, d \mid c=a^{-1} b a, d=a^{-1} c a,\left[a b^{-1}, c\right],\left[a b^{-1}, d\right]\right\rangle, \\
F=\left\langle a, b, c, d, e \mid c=a^{-1} b a, d=a^{-1} c a, e=a b^{-1},[e, c],[e, d]\right\rangle .
\end{gathered}
$$

Note that the generators $a, b, c, d$ above are usually denoted $x_{0}, x_{1}, x_{2}, x_{3}$ respectively in the Thompson's group literature.

We display the mean length computed from our Markov chain for these three presentations in Figure 8. In all cases we also saw no indication of a singularity at the amenable values of $\beta=1 / 3, \beta=1 / 7$ and $\beta=1 / 9$ respectively. We also repeated the error-analysis that was done for $\widetilde{G}$ above - see Figure 9 . Again we saw no indication of a singularity present in these statistics at the amenable value of $\beta$. We have made rough estimates of the location of the dominant singularity of the 


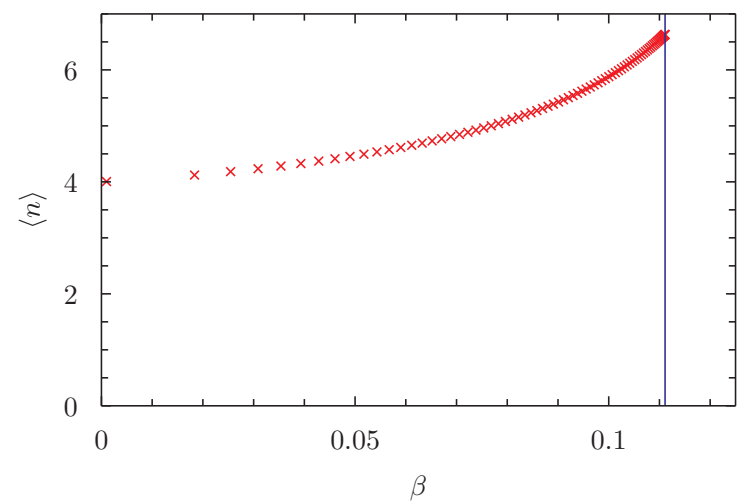

(A) Mean length with $\alpha=1$.

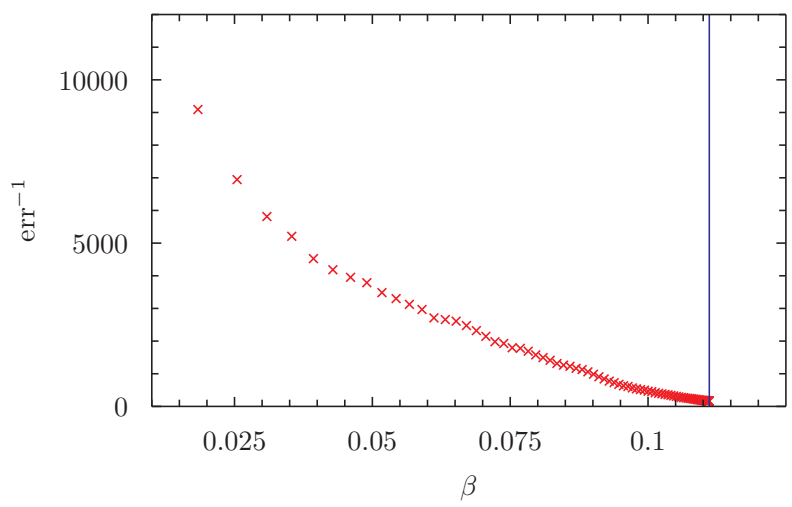

(в) err $^{-1}$ with $\alpha=1$.

Figure 7. (A) The mean length of words plotted against $\beta$ for the presentation of group $\widetilde{G}$ in equation 3.14 . (B) The reciprocal of the estimated error against $\beta$. Notice that as $\beta \rightarrow 1 / 9$ the error begins to diverge.

cogrowth series by estimating where the reciprocal of the observed error goes to zero. The data from these presentations were easier to analyse than that from $\widetilde{G}$ and because of this we were able to obtain estimates with tighter error bars. Our analysis gives

$$
\beta_{c}=0.395 \pm 0.005,0.172 \pm 0.002 \text { and } 0.134 \pm 0.004
$$

for the three presentations. These imply cogrowths of approximately $2.53 \pm 0.03,5.81 \pm$ 0.07 and $7.4 \pm 0.2$, all of which are well below the amenable values of 3,7 and 9 .

Of course, these estimates do not constitute a proof that Thompson's group is non-amenable. However, they are stronger numerical evidence than any previous work (such as [1, 6] and [16]). As is the case with almost any numerical experiment, one cannot rule out the presence of particular pathalogical behaviours in Thompson's group that distort the behaviour of the chain and so the numerical data. 


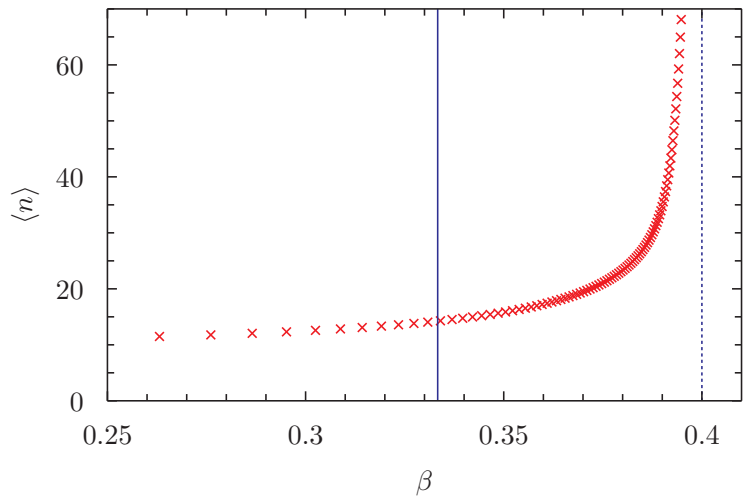

(A) Standard presentation 3.15 for $F$ sampled with $\alpha=2$

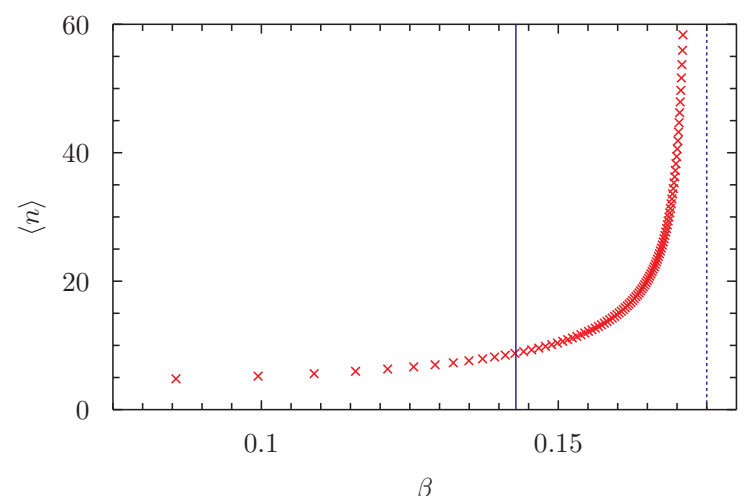

(в) Presentation 3.16 for $F$ sampled with $\alpha=2$

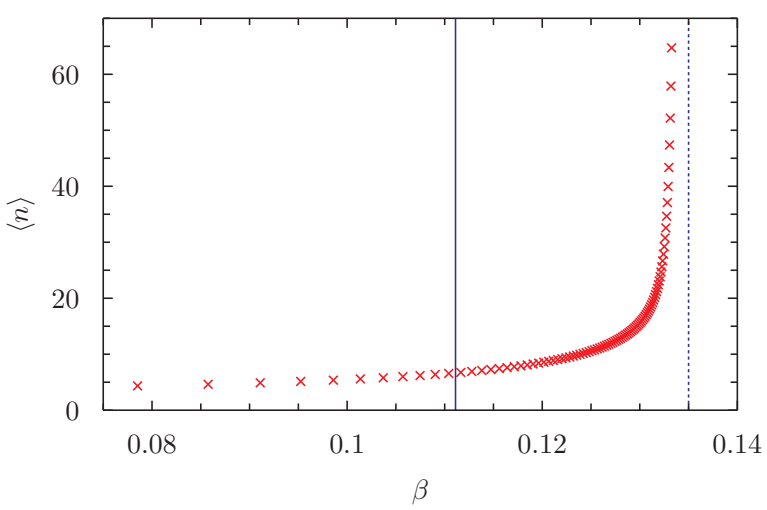

(c) Presentation 3.17 for $F$ sampled with $\alpha=1$

Figure 8. Mean length of freely reduced trivial words in Thompson's group $F$ at different values of $\beta$. The solid blue lines indicate the reciprocal of the cogrowth of amenable groups with $k$ generators $\beta_{c}=1 /(2 k-1)$. The dashed blue lines indicate the approximate location of the vertical asymptote. In each case, we see that the mean length of trivial words is finite for $\beta$-values slightly above $\beta_{c}$. 


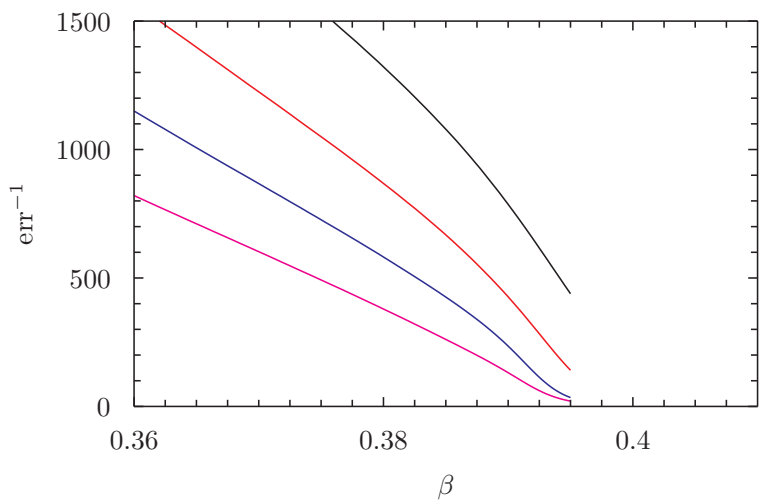

(A) Standard presentation 3.15 for $F$ sampled with $\alpha=0,1,2,3$.

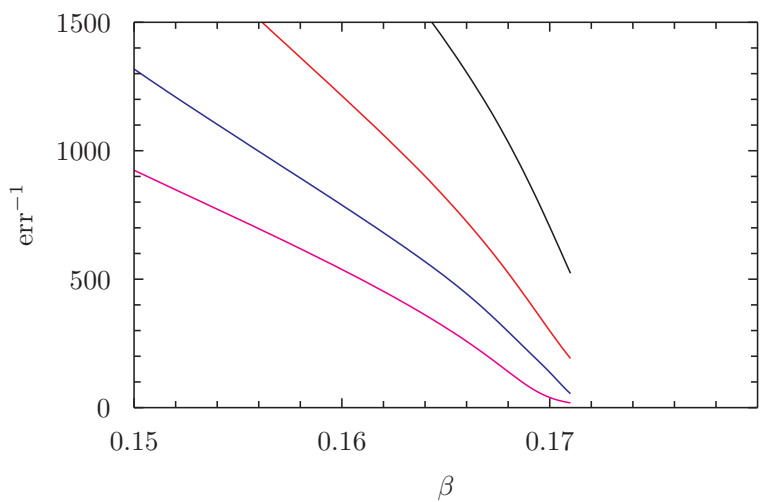

(B) Presentation 3.16 for $F$ sampled with $\alpha=0,1,2,3$.

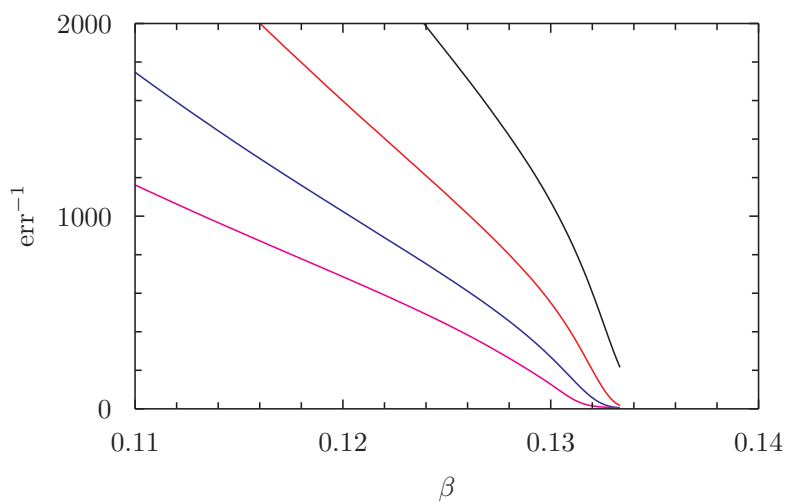

(c) Presentation 3.17 for $F$ sampled with $\alpha=0,1,2,3$.

Figure 9. The reciprocal of the estimated standard error of the mean length as a function of $\beta$ for the three presentations of Thompson's group. In each plot we show 4 curves corresponding to simulations at $\alpha=0,1,2,3$ (anti-clockwise from top). Extrapolating these curves leads to estimates of $\beta_{c}$ of $0.395 \pm 0.005$, $0.172 \pm 0.002,0.134 \pm 0.004$. These are all well above the values of amenable groups. 


\section{Conclusions}

We have introduced a novel Markov chain which samples trivial words from finitely presented groups. Since this chain operates on the state space of trivial words rather than on the Cayley graph, it is quite different from previous studies of random walks on groups. We have shown that the Markov chain converges to the stationary distribution $\pi$ and so asymptotically samples from it. Further, $\pi$ is a stretched Boltzmann distribution related to the cogrowth series of the presentation and so statistics collected from the chain inform us about the cogrowth of the group.

We have implemented the chain for presentations of both amenable and nonamenable groups for which the cogrowth series is known exactly. In these cases we observe excellent agreement between statistics collected from our chain and exact results. We have also implemented the chain for presentations of groups for which little is known about the cogrowth series. In the case of the basilica group (or more precisely a finitely presented group into which the basilica group embeds), our results are consistent with the amenability of the group. On the other hand, our results for Thompson's group $F$ suggest that it is not amenable.

In cases where the cogrowths series is known exactly (or can be computed to arbitrary precision) we observed that the mean length statistic generated by our chains converged quickly to the correct value. This behaviour held for both amenable and non-amenable groups.

As is the case with any numerical experiment we cannot rule out the presence of pathologies influencing our results. This raises two obvious questions which lie beyond this present work: how can we determine the rate at which the Markov chain convergences to the stationary distribution; and how can we analyse statistics from the chain to obtain precise estimates of the asymptotic behaviour of the cogrowth function. Both of these questions have strong implications for numerical tests of the amenability of a group, and we intend to pursue them in future work.

\section{ACKNOWLEDGEMENTS}

The authors thank Sean Cleary, Tony Guttmann and Stu Whittington for helpful discussions about this work. Much of the numerical work was run on the Westgrid computer cluster and the authors thank Westgrid for their support. This research was supported by the Australian Research Council (ARC), the the Natural Sciences and Engineering Research Council of Canada (NSERC), and Perimeter Institute for Theoretical Physics. Research at Perimeter Institute is supported by the Government of Canada through Industry Canada and by the Province of Ontario through the Ministry of Economic Development and Innovation.

\section{REFERENCES}

[1] G. N. Arzhantseva, V. S. Guba, M. Lustig, and J. Préaux. Testing Cayley graph densities. Ann. Math. Blaise Pascal, 15(2):233-286, 2008.

[2] L. Bartholdi, V. A. Kaimanovich, and V. V. Nekrashevych. On amenability of automata groups. Duke Math. J., 154(3):575-598, 2010.

[3] L. Bartholdi and B. Virág. Amenability via random walks. Duke Math. J., 130(1):39-56, 2005.

[4] L. Bartholdi and W. Woess. Spectral computations on lamplighter groups and Diestel-Leader graphs. J. Fourier Anal. Appl., 11(2):175-202, 2005.

[5] J. M. Belk and K. S. Brown. Forest diagrams for elements of Thompson's group F. Internat. J. Algebra Comput., 15(5-6):815-850, 2005. 
[6] J. Burillo, S. Cleary, and B. Wiest. Computational explorations in Thompson's group F. In Geometric group theory, Trends Math., pages 21-35. Birkhäuser, Basel, 2007.

[7] J. M. Cohen. Cogrowth and amenability of discrete groups. J. Funct. Anal., 48(3):301-309, 1982.

[8] P. Diaconis and L. Saloff-Coste. Comparison techniques for random walk on finite groups. Ann. Probab., 21(4):2131-2156, 1993.

[9] P. Diaconis and L. Saloff-Coste. Comparison theorems for reversible Markov chains. Ann. Appl. Probab., 3(3):696-730, 1993.

[10] P. Diaconis and L. Saloff-Coste. Moderate growth and random walk on finite groups. Geom. Funct. Anal., 4(1):1-36, 1994.

[11] P. Diaconis and L. Saloff-Coste. Random walks on finite groups: a survey of analytic techniques. In Probability measures on groups and related structures, XI (Oberwolfach, 1994), pages 44-75. World Sci. Publ., River Edge, NJ, 1995.

[12] P. Diaconis and L. Saloff-Coste. Walks on generating sets of groups. Invent. Math., 134(2):251-299, 1998.

[13] K. Dykema. Symmetric random walks on certain amalgamated free product groups. In Topological and asymptotic aspects of group theory, volume 394 of Contemp. Math., pages 87-99. Amer. Math. Soc., Providence, RI, 2006.

[14] K. Dykema and D. Redelmeier. Lower bounds for the spectral radii of adjacency operators on Baumslag-Solitar groups. Preprint, arXiv:1006.0556, 2010.

[15] M. Elder, A. Rechnitzer, E. J. Janse van Rensburg, and T. Wong. The cogrowth series for $\operatorname{BS}(n, n)$ is D-finite. Preprint, arXiv:1309.4184, 2013.

[16] M. Elder, A. Rechnitzer, and T. Wong. On the cogrowth of Thompson's group F. Groups Complex. Cryptol., 4(2):301-320, 2012.

[17] C. J. Geyer and E. A. Thompson. Annealing Markov chain Monte Carlo with applications to ancestral inference. Journal of the American Statistical Association, pages 909-920, 1995.

[18] R. I. Grigorchuk. Symmetrical random walks on discrete groups. In Multicomponent random systems, volume 6 of Adv. Probab. Related Topics, pages 285-325. Dekker, New York, 1980.

[19] R. I. Grigorchuk and Żuk A. On a torsion-free weakly branch group defined by a three state automaton. In International conference on geometric and combinatorial methods in group theory and semigroup theory, volume 12 of Internat. J. Algebra Comput., pages 223-246. World Scientific, Singapore, 2002.

[20] E. J. Janse van Rensburg. Monte Carlo methods for the self-avoiding walk. Journal of Physics A: Mathematical and Theoretical, 42:323001, 2009.

[21] S. Karlin and H.E. Taylor. A first course in stochastic processes. Elsevier, 1975.

[22] D. Kouksov. On rationality of the cogrowth series. Proc. Amer. Math. Soc., 126(10):2845$2847,1998$.

[23] D. Kouksov. Cogrowth series of free products of finite and free groups. Glasg. Math. J., 41(1):19-31, 1999.

[24] S. P. Lalley. The weak/strong survival transition on trees and nonamenable graphs. In International Congress of Mathematicians. Vol. III, pages 637-647. Eur. Math. Soc., Zürich, 2006.

[25] R. C. Lyndon and P. E. Schupp. Combinatorial group theory. Springer-Verlag, Berlin, 1977. Ergebnisse der Mathematik und ihrer Grenzgebiete, Band 89.

[26] N. Madras and G. Slade. The self-avoiding walk. Probability and its Applications. Birkhäuser Boston Inc., Boston, MA, 1993.

[27] A. Mann. How groups grow, volume 395 of London Mathematical Society Lecture Note Series. Cambridge University Press, Cambridge, 2012.

[28] N. Metropolis, A. W. Rosenbluth, M. N. Rosenbluth, A. H. Teller, and E. Teller. Equation of State Calculations by Fast Computing Machines. Journal of Chemical Physics, 21:1087-1092, June 1953

[29] M. Mitzenmacher and E. Upfal. Probability and computing: Randomized algorithms and probabilistic analysis. Cambridge University Press, 2005.

[30] J. T. Moore. Fast growth in the Følner function for Thompson's group F. Groups Geom. Dyn., 7(3):633-651, 2013.

[31] T. Nagnibeda. An upper bound for the spectral radius of a random walk on surface groups. Zap. Nauchn. Sem. S.-Peterburg. Otdel. Mat. Inst. Steklov. (POMI), 240(Teor. Predst. Din. Sist. Komb. i Algoritm. Metody. 2):154-165, 293-294, 1997. 
[32] T. Nagnibeda. Random walks, spectral radii, and Ramanujan graphs. In Random walks and geometry, pages 487-500. Walter de Gruyter GmbH \& Co. KG, Berlin, 2004.

[33] R. Ortner and W. Woess. Non-backtracking random walks and cogrowth of graphs. Canad. J. Math., 59(4):828-844, 2007.

[34] J.S. Rosenthal. A first look at rigorous probability theory. World Scientific, 2006.

[35] M. C. Tesi, E. J. Janse van Rensburg, E. Orlandini, and S. G. Whittington. Monte Carlo study of the interacting self-avoiding walk model in three dimensions. Journal of Statistical Physics, 82(1):155-181, 1996.

[36] S. Wagon. The Banach-Tarski paradox. Cambridge University Press, Cambridge, 1993. With a foreword by Jan Mycielski, Corrected reprint of the 1985 original.

[37] W. Woess. Cogrowth of groups and simple random walks. Arch. Math. (Basel), 41(4):363-370, 1983.

School of Mathematical \& Physical Sciences, The University of Newcastle, Callaghan, New South Wales, Australia

E-mail address: murray.elder@newcastle.edu.au

Department of Mathematics, University of British Columbia, Vancouver, British Columbia, Canada

E-mail address: andrewr@math.ubc.ca

York University, Toronto, Ontario, Canada

E-mail address: rensburg@mathstat.yorku.ca 